\title{
Study of Moisture Content Automatic Detection Based on Single Chip Microcomputer
}

\author{
Liu Caixia ${ }^{1, a^{*}}$, Li Zhuanye ${ }^{2}$, Yan Qingzhi ${ }^{2}$ and Hou Yanyan ${ }^{1}$ \\ ${ }^{1}$ Department of Information Science and Engineering, Zaozhuang University. China \\ ${ }^{2}$ Ltd. Weifang Liu He Electricity, Weifang, China \\ acxsqz@126.com
}

\begin{abstract}
Keywords: moisture content; crude oil; capacitance sensor; dielectric constant; single chip microcomputer
\end{abstract}

\begin{abstract}
Precise and high efficiency of moisture content detection is important to the oil production and management in the oil chemical industry. In this paper, a new technology on moisture content automatic detection based on single chip microcomputer is proposed. This system can accurately measure the moisture content automatically of the crude oil oilcan in department of oil and chemical.
\end{abstract}

\section{Introduction}

In the oil chemical industry, the moisture content of the oil is one of the most measure parameters in the oil production and processing[1-2]. The preciseness and high efficiency of moisture content detection is important to the oil production and management[3-4]. Especially, the correct and timely moisture content data can reflect whether the oil can works well or not. This can instruct production and adopt efficient measures, improve arts and craft to rationally exploit energy, and avoid the waste of man power, material resources.

Now, the moisture content in crude oil storage tank is mainly depend on manpower sampling and distillation testing in some oil chemical industries. This method has the disadvantage of little sampling, difficult sampling, data lagging and influenced by environment and so on. What's more, mass of electric power and oil are wasted. Scholars at home and abroad have put forward a lot of methods, but most of them are too complicated [5-7].

In this paper, a new technology on moisture content automatic detection based on single chip is proposed. The computer put out order, control unit of instrument received order and controlled the motor running which brought along the bobbin to make the sensor up and down. When the sensor arrived at the bottom the oilcan, it sent signal and then the motor reverse running and the sensor was brought back. When the sensor arrived at the top, it set signal again and the motor stopped and one measurement is completed. The collection data was set back to computer and the gross weight of oil was gotten. This system can accurately measure the moisture content automatically of the crude oil oilcan in department of oil and chemical.

\section{A New Technology Of Moisture Content Automatic Detection}

The sensitive capacitance sensor probe is a changed dielectric constant form capacitance sensor. This kind sensor is sensitive to dielectric constant. At the same time the dielectric constant of the crude oil containing water is changed with the change of the moisture content. The crude oil can be taken as the mixture of pure oil and pure water if the impurity is neglected. The pure oil is non-polarity electrolyte and dielectric constant is at 2.3 about. The pure water is polarity electrolyte and the dielectric constant is at 80 about. By all appearances, the change of moisture content in crude oil will remarkably influence the change of crude oil dielectric constant. When the sensitive probe is set into the crude oil, the change of water content oil will convert to the change of capacitance of sensitive probe, and the capacitance sensor sends an electric signal of moisture content, then the moisture content is detected. 
The Working Principle Of Capacitance Sensor. As the Fig.1 shows, the left of circuitry is stable high frequency oscillator and the right is resonance circuit. By sensor coupling, the surge voltage is obtained from stable high frequency oscillator. The capacitance $\mathrm{C}$ of sensitive capacitance sensor probe is one part of tune capacitance in resonance circuit. The capacitance of resonance circuit is changed and that makes the inherent frequency of resonance circuit changed when the moisture content changed. When the inherent frequency of resonance circuit and the frequency of high frequency oscillator are greatly different, loop is in the mistuning state and the voltage value is low after demodulation. If these two frequencies are equal, loop is in the resonant state and the voltage value is high after demodulation. In fact, the inherent frequency of resonance circuit is changed as the moisture content of crude oil. When the probe is set in the pure water, the loop is in the resonant state and the voltage value is high after demodulation. When the probe is set in the pure oil, loop is in the mistuning state and the voltage value is low after demodulation. When the probe is set in the oil containing some water, loop is in the state which is not totally mistuning and not totally syntonic and the voltage value is between above two values. So the moisture content in the probe position can be detected according to the demodulation voltage value.

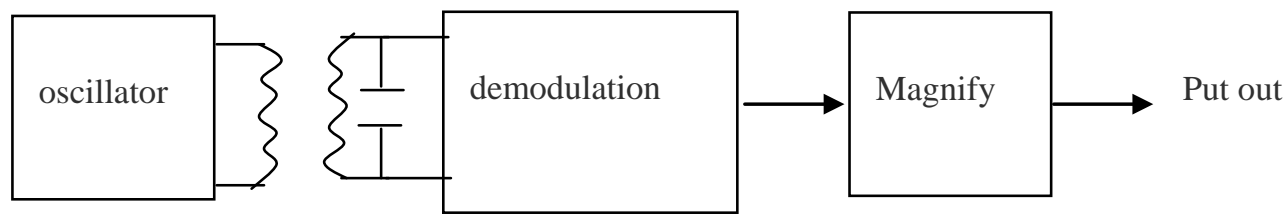

Fig. 1 Capacity sensor principle picture

The Calculation On Surge Frequency And The Capacitance Of Sensitive Capacitance Sensor Probe. The surge frequency of oscillator is gotten according to the impedance property of the detected medium. The impedance property of oil and water is changed as the surge frequency. The experiment proved that impedance property difference of oil and water achieved maximum when surge frequency at $10 \mathrm{MHz}$ about. So we can take its surge frequency as $10 \mathrm{MHz}$.

$$
\frac{2 \pi L \varepsilon_{0} \varepsilon_{r} \varepsilon_{r r}}{\varepsilon_{r} l n \frac{r_{A}}{r_{B}}+\varepsilon_{x r} l n \frac{r_{B}}{r_{A}}}
$$

The parameters are as follows:

$L$ : the length of probe.

$\varepsilon_{\mathrm{xr}}$ : the relatively dielectric constant of the detected liquid.

$r_{\mathrm{A}}$ : the inner radius of exterior conductor.

$\varepsilon_{\mathrm{r}}$ : the relatively dielectric constant of the insulated cover material.

$r_{\mathrm{B}}$ : the exterior radius of the insulated cover material.

$\varepsilon_{0}$ : The vacuum dielectric constant.

The Calculation Of Sensor Position. The bobbin drives the sensor up and down in the oilcan controlled by stepping motor. The position of the sensor in the oilcan can be detailed and accurate calculated by the amount of pulses of stepping motor, the radius of bobbin and the layer difference of every layer.

\section{The Working Principle Of The Whole System.}

As shown in figure 2, computer set measure order, the 89c52 of control equipment accept order and control the running of motor to measure. The sensor continually set back the collected data. The moisture content and temperature of the corresponding highness are gotten according to the data set back by sensor, and the sensor position is gotten by the amount of pulses of stepping motor. the temperature of moisture content is compensated by the computer, and then the gross weight of oil is calculated according to the diameter of oil tank, the moisture content and the relatively highness. The precision of oil weight is high by revising special curve and temperature compensating curve as well as a large mount of sample. 


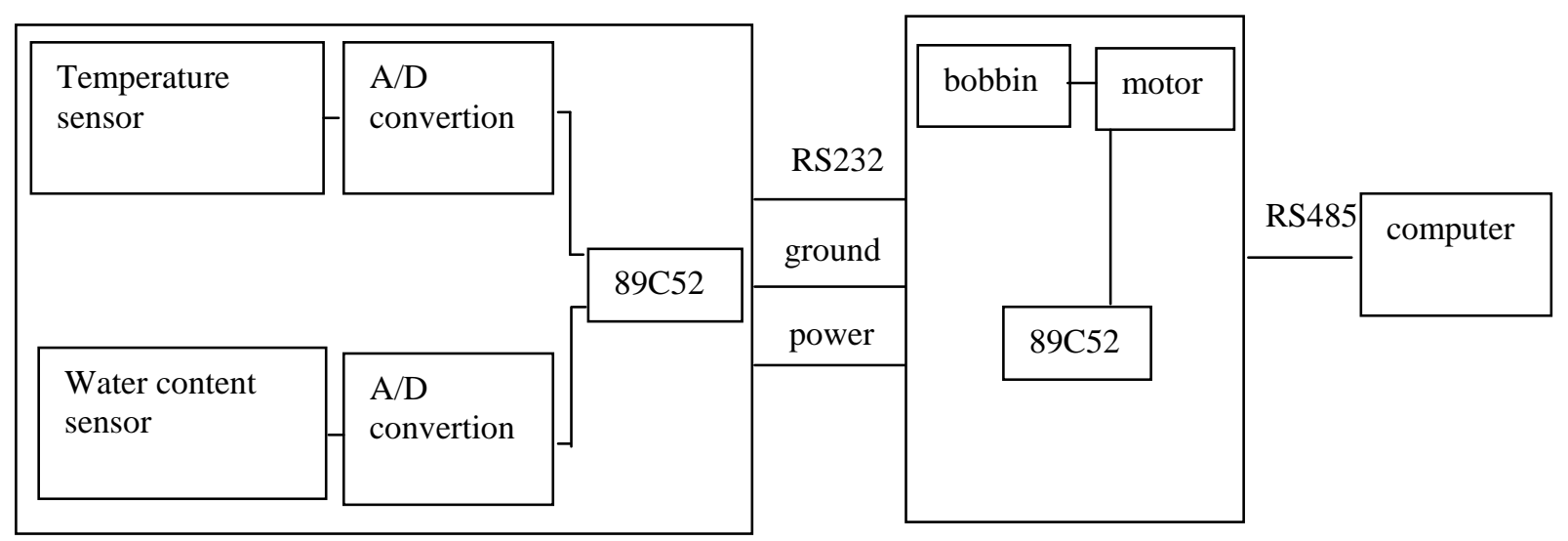

Fig. 2 The working principle of the whole system

The finally measurement extent and precision are as table 1.

Table 1 . The finally measurement extent and precision

\begin{tabular}{cc}
\hline measurement extent & precision \\
\hline Liquid highness $\leq 20 \mathrm{~m}$ & precision $\leq \pm 2 \mathrm{~mm}$ \\
Crude oil interface $\leq 20 \mathrm{~m}$ & precision $\leq \pm 10 \mathrm{~mm}$ \\
Mud highness $\leq 10 \mathrm{~m}$ & precision $\leq \pm 5 \mathrm{~mm}$ \\
Moisture content $\leq 0-99.99 \%$ & precision $\leq \pm 1.5 \%$ \\
crudeoiltemperature $\leq 0-100^{\circ} \mathrm{C}$ & precision $\leq \pm 0.5^{\circ} \mathrm{C}$ \\
\hline
\end{tabular}

\section{Conclusion}

This system realized the acquisition of multiple sets of data only by one measurement, including tank liquid level, oil level, sludge height, the moisture content at each height of tank, oil heavy measurement. The system is controlled by single chip and the computer. The instrument can be adiusted directly and remotely set up. What is more, it is easy to use and control.

\section{References}

[1] Hou Guopei, Shi Jianshan, Wang Yutian. Study on Instrument for Measuring Water Cut in Oil. Chinese Journal of Sensors and Actuators. 2000, (01), pp:44-48.

[2]Tian Jingjing.Research of the Crude Oil Water-Rate measurement by r-ray.Tianjin University,2005.

[3]Pi Fumin. Design of the Detection System for Water Ratio in Oil, XIDIAN UNIVERSITY,2014.

[4]Ji Na.Study of the On-line Measurement System of the Water Content in crude oil. Hebei University.2010.

[5]Mu Lei. The design of a high-temperature experimental model testing the moisture content.Daqing Petroleum Institute, 2009.

[6]Wu Guozhong, Zheng Xiaolan,Wu Junxue, Li Haojie.Processing of water cut of crude oil data based on back propagation artificial neural networks. Petrochemical industry application.2009,(04),pp:35-37. 
[7]Yang Chuanfa. Research on measurement of the moisture contents in the crude oil by microwave transmission. Shenyang University of Technology,2010. 